\section{Prostatektomie: Blasenhals nicht zu eng machen!}

\author{
Im Zuge der blasenhalserhaltenden Prostatektomie ist es offenbar nicht sinn- \\ voll, einen besonders engen Blasenhals anzustreben. In puncto Inkontinenz \\ zeigte sich in einer Studie kein Vorteil bei geringen Durchmessern.
}

B ei der Prostatektomie wird heute zunehmend Wert auf den Erhalt des Blasenhalses gelegt. Dadurch sollen die Patienten postoperativ schneller wieder eine möglichst optimale Kontinenz erlangen. Auf die Stärke, sprich den Durchmesser des verbleibenden Blasenhalses scheint es dabei jedoch nicht so sehr anzukommen.

Ein Team um Mark D. Tyson, USA, hat 107 Patienten mit Prostatakarzinom prospektiv nachbeobachtet, die sich einer robotergestützten laparoskopischen radikalen Prostatektomie (RALP) unterzogen hatten. Als primärer Endpunkt diente den Forschern die subjektive Inkontinenzrate, angegeben als Wert im EPIC(Expanded Prostate Cancer Index Composite)-Score. Der Gesamtscore umfasst 100 Punkte, wobei ein höherer Wert für eine bessere Blasenfunktion steht. Die Blasenhalsdicke wurde intraoperativ gemessen, indem man vor der Ausführung der vesikourethralen Anastomose durch eine zusätzliche Öffnung ein Lineal einbrachte und so den größten Durchmesser ermittelte. Dieser betrug im Mittel $13 \mathrm{~mm}$.

Sechs sowie zwölf Wochen nach dem Eingriff wurden die Patienten gebeten, einen Fragebogen zur Blasenfunktion (EPIC-Score) auszufüllen. Beide Male zeigte sich kein nennenswerter Einfluss der Blasenhalsdicke auf die Inkontinenz. Nach sechs Wochen lag der Beta-Koeffizient - die geschätzte Differenz im Blasenhalsdurchmesser zwischen Patienten im höchsten versus Patienten im nied- rigsten Perzentil - bei 0,88. Das höchste Perzentil entsprach $18 \mathrm{~mm}$, das niedrigste $7 \mathrm{~mm}$. Nach zwölf Wochen betrug der Beta-Koeffizient 5,80. Beide Werte waren nicht signifikant. In drei Fällen fand sich ein tumorzell-positiver Schnittrand am Blasenhals: Die Blasenhalsstärken dieser Patienten betrugen 5, 7 und $15 \mathrm{~mm}$.

Fazit: „Alle Bemühungen, den Blasenhalsdurchmesser so klein wie möglich zu halten, haben die frühen Kontinenzergebnisse nicht maßgeblich beeinflusst", so Tyson und sein Team. Damit sei jedoch nicht gesagt, dass die blasenhalsschonende Technik per se unwirksam sei. In der vorliegenden Studie habe man lediglich den Effekt unterschiedlicher Blasenhalsstärken innerhalb der blasenhalserhaltenden Prostatektomie untersucht und nicht das Verfahren an sich bewertet.

Dr. Elke Oberhofer

Tyson MD et al. The null effect of bladder neck size on incontinence outcomes after radical prostatectomy. J Urol. 2017; https://doi. org/10.1016/j.juro.2017.06.084.

\title{
Zytostatikum stabilisiert Erfolg der Harnröhrenschlitzung
}

\section{Das hohe Rezidivrisiko nach Urethrotomia interna lässt sich vermutlich sen- ken, wenn dabei Mitomycin C in die Läsion injiziert wird.}

$\mathrm{D}^{\mathrm{s}}$ er Stellenwert der Urethrotomia interna wird durch das hohe Risiko für längere Rezidivengen limitiert. Michael R. Farrell und Kollegen haben versucht, den Erfolg der Intervention durch Injektion von Mitomycin C zu steigern. Durch das Zytostatikum sollte eine weitere Fibrosierung vor allem im angrenzenden gesunden Gewebe verhindert werden.

Die 44 Patienten der Studie hatten nach mindestens einer vorausgegangenen endoskopischen oder offen chirurgischen Therapie erneut eine symptomatische Striktur der bulbären/bulbär-membranösen Urethra mit einer Länge von median $2 \mathrm{~cm}$ entwickelt. Bei 18 Patienten war die Striktur durch Brachytherapie und/oder externe Bestrahlung induziert.

Bei der Urethrotomie unter Sicht wurde die narbige Engstelle bis zum gesun- den Gewebe mit dem kalten Messer in den Positionen 12, 3 und 9 Uhr inzidiert, dann wurden intraläsional $4 \mathrm{mg}$ Mitomycin C injiziert. Nach dem Eingriff hatte der maximale Harnfluss in der Uroflowmetrie signifikant zugenommen, sowohl in der Gesamtgruppe als auch bei den Patienten mit nicht durch Radiotherapie induzierter Striktur. Bei den Patienten mit Strahlungsschaden war keine Verbesserung festzustellen. Das Restharnvolumen hatte in keiner Patientengruppe abgenommen. 33 der 44 Patienten (75 \%) benötigten während der Nachbeobachtungszeit von median 26 Monaten keine weitere chirurgische Behandlung. Bei den verbleibenden elf Patienten vergingen bis zum Rezidiv median 10,7 Monate. Ein Rezidiv war definiert als Striktur, die mit einem flexiblen 16-Charrière -Zysto- skop nicht passierbar war, und/oder als obstruktive Miktionsbeschwerden, die weitere Maßnahmen erforderten.

Fazit: Laut Farrell et al. handelt es sich bei der Urethrotomia interna unter Sicht mit Mitomycin $\mathrm{C}$ und anschließender sauberer intermittierender Katheterisierung ,um eine sichere, effektive und breit verfügbare Methode zum Management komplexer wiederkehrender kurzer $(<3$ $\mathrm{cm}$ ) bulbärer und bulbär-membranöser Urethrastrikturen“. Wenn Begleiterkrankungen oder der Patientenwunsch einem offen operativen Verfahren entgegenstünden, könne die Schlitzung mit Mitomycin-C-Applikation in Erwägung gezogen werden. Bei Patienten mit Lichen sclerosus oder einer Strikturlänge über $3 \mathrm{~cm}$ raten die Urologen allerdings ausdrücklich von der endoskopischen Behandlung $\mathrm{ab}$.

Dr. Beate Schumacher

Farrell MR, Lawrenz C, Levine LA. Internal urethrotomy with intralesional Mitomycin C: an effective option for endoscopic management of recurrent bulbar and bulbomembranous urethral strictures. Urology. 2017; https://doi. org/10.1016/j.urology.2017.07.017. 